\title{
Small dense low density lipoprotein- cholesterol and cholesterol ratios to predict arterial stiffness progression in normotensive subjects over a 5 -year period
}

Gang $\mathrm{Li}^{1,2+}$, Hui-kun $\mathrm{Wu}^{3,4+}$, Xiao-wei $\mathrm{Wu}^{5^{*}}$, Zhe Cao ${ }^{6}$, Yuan-chao $\mathrm{Tu}^{2^{*}}$, Yi Ma ${ }^{1}$, Wei-qing Wang ${ }^{1}$, Jian Cheng $^{7}$ and Zi-hua Zhou ${ }^{8}$

\begin{abstract}
Background: Small dense low density lipoprotein-cholesterol (sdLDL-C), cholesterol ratios and carotid-femoral pulse wave velocity (cf-PWV) impart risk for all-cause morbidity and mortality independently of conventional cardiovascular disease (CVD) risk factors. This study was designed to identify feasible indicators for predicting arterial stiffness progression.

Methods: We followed up 816 normotensive participants without diabetes or CVD for nearly 5.0 years. Cholesterol parameters, ratios and other clinical and laboratory data were collected at baseline. cf-PWV were measured at baseline and the end of follow-up.

Results: PWW progression subjects had higher levels of PWW parameters, sdLDL-C and TG/HDL-C ratio. sdLDL-C and TG/ HDL-C were significantly correlated with all PWV parameters. Multiple regression models showed that sdLDL-C was closely associated with follow-up PWV $(\beta=0.222, p<0.001)$ and $\triangle \mathrm{PWV}(\beta=0.275, p<0.001)$. TG/HDL-C was only one cholesterol ratios that associated with all PWV parameters. sdLDL-C (OR $=2.070,95 \% \mathrm{Cl}: 1.162$ to $3.688, p=0.014)$ and $\mathrm{TG} / \mathrm{HDL}-\mathrm{C}(\mathrm{OR}=1.355,95 \% \mathrm{Cl}: 1.136$ to $1.617, p=0.001)$ could significantly determine the progression of PWV after correction for covariates. High sd-LDL-C quantiles subjects were more likely to develop arterial stiffness progression than low quantiles (Tertiles 3 vs Tertiles1, RR=2.867, 95\%Cl: 1.106 to 7.434, $p=0.03$ ).
\end{abstract}

Conclusion: We founded that sdLDL-C and TG/HDL-C ratio can independently predict arterial stiffness progression in normotensive subjects, and high level sdLDL-C and TG/HDL-C ratio were associated with a higher risk of arterial stiffness.

Keywords: sdLDL-C, Cholesterol ratios, Arterial stiffness, Normotensive

\section{Background}

Arterial stiffness is aggravated with age, which is extensively associated with atherosclerotic vascular diseases. There are several ways of determining arterial stiffness, among which carotid-femoral pulse wave velocity (cf-PWV) is currently considered the gold standard [1]. cf-PWV directly reflects, and has the best clinical correlation to, aortic stiffness [1]. Epidemiological studies have confirmed that cf-PWV is a

\footnotetext{
*Correspondence: wuxiaowei119@gmail.com; tuyuanchao@hbhtcm.com ${ }^{\dagger}$ Equal contributors

${ }^{5}$ Department of Thoracic Surgery, TongJi Hospital, TongJi Medical College, Huazhong University of Science and Technology, Wuhan, China ${ }^{2}$ Hubei Province Academy of Traditional Chinese Medicine, Wuhan, China

Full list of author information is available at the end of the article
}

predictor of cardiovascular events and is one of the few indicators of arterial stiffness directly related to cardiovascular morbidity and mortality [2-4].

Several variables affect arterial stiffness, including age gender, blood pressure, high heart rate and life style, a variety of cardiovascular risk factors as well as genetic and systemic inflammation [5-8].Blood lipid parameters such as total cholesterol (TC), triglyceride (TG) and low-density lipoprotein cholesterol (LDL-C) are cardiovascular risk factors associated with atherosclerosis, but they cannot constantly predict arterial stiffness progression [9]. Recently, small dense low density lipoproteincholesterol (sdLDL-C) was regarded as one of the 
lipoprotein risk factors for coronary heart disease (CHD) and as the best marker of carotid atherosclerosis $[10,11]$. However, little is known about the association between sdLDL-C and cf-PWV, especially in normotensive population. Cholesterol ratios are commonly used to reflect cardiovascular risk. A number of studies have shown that some of cholesterol ratios are associated with arteriosclerosis indicators such as carotid intima media thickness (CIMT) [12] and cardio-ankle vascular index (CAVI) [13]. In the Japanese diabetic population, high TG/HDL-C is independently associated with CAVI [14]. The TC/HDL-C ratio is highly correlated with other cardiovascular risk factors, including CIMT, high body mass index (BMI) and metabolic syndrome [12, 15]. However, the relationship between cholesterol ratios and cf-PWV, which is the gold standard to reflect arterial stiffness, has not been studied in a cohort with normal blood pressure. Additionally, whether or not these ratios can predict early arterial stiffness progression is unknown.

This study aimed to explore the relationship between sdLDL-C, cholesterol ratios and arterial stiffness progression which measured by the gold standard of cfPWV. We followed up a normotensive population to identify an optimal indicator for predicting early arterial stiffness progression.

\section{Methods}

\section{Study population}

From September 2010 through March 2011, 1148 consecutive normotensive subjects without known cardiovascular disease according to WHO guidelines were selected for study. Subjects were invited to participate in a study for evaluation of cf-PWV. The inclusion criteria included normal blood pressure (systolic blood pressure $[\mathrm{SBP}]<140$ and diastolic blood pressure $[\mathrm{DBP}]<90 \mathrm{~mm}$ $\mathrm{Hg}$ ) and age of 18 years or higher. Patients with the following conditions were excluded: history of heart failure or cardiomyopathy, coronary heart disease, resting electrocardiogram (ECG) abnormalities ( $Q$ waves or left bundle branch block), cerebrovascular or peripheral artery disease and diabetes. Five years later, the subjects were invited to undergo the same arterial examinations. Overall, 816 subjects ( $71 \%$ of those invited) had their second visit from October 2015 through April 2016. Among the 332 subjects who did not have follow-up measurements, 198 lost contacts, 54 refused, and 20 did not return for unknown reasons. All participants provided written informed consent at the first visit.

In the present study, family income was classified as <30,000, 30,000-100,000, and >100,000 CNY/y. Educational level was categorized as low (no schooling, incomplete primary education, and primary education), middle (3 or 4 years of secondary education), and high (college and university education). Physical activity was classified into three groups using methods presented elsewhere [16]. We defined alcohol consumption as the weekly consumption of beer, wine and hard liquor. Current drinking was defined as alcohol consumption $\leq 8 \mathrm{ml}$ per week according to the definition from the National Institute on Alcohol Abuse and Alcoholism [17]. We defined smokers as people who smoked at least one cigarette per day and continued for at least 1 year. We asked whether they currently smoked (Do you smoke currently?).

\section{Clinical investigations}

Blood samples were collected in the morning after overnight fasting for at least $12 \mathrm{~h}$. Blood was drawn into plastic tubes containing EDTA-2Na, and chilled in ice. Plasma was obtained by centrifugation at $4{ }^{\circ} \mathrm{C}$, and kept at $0-4{ }^{\circ} \mathrm{C}$ until assays. All measurements were performed within 6 h. Fasting blood glucose(FBG), liver enzyme levels, serum lipid profiles, including TG, TC, LDL-C)and HDL-C were determined on a Olympus AU600 analyzer (Olympus, Tokyo, Japan). sdLDL-C was measured by the method of Hirano et al. [18] with minor modification [13] using the commercially available assay kit (sdLDL SEIKEN, Denka Seiken Co., Ltd., Tokyo, Japan). Apolipoproteins (apo) A-1, and B were measured by immunoturbidimetry (Daiichi Pure Chemicals Co., Ltd.,Tokyo). The ELISA technique was used to measure levels of high-sensitivity $C$ reaction protein (hs-CRP, Immunodiagnostik Ag). The sensitivity of the assay was $0.05 \pm 0.007$. The cytokines interleukin6 (IL-6) and tumor necrosis factor- $\alpha$ (TNF-á, R \& D Systems Ltd.) were measured by ELISA in duplicates. The TNF-á and IL- 6 assays detected concentrations down to 0.32 and $0.11 \mathrm{pg} / \mathrm{ml}$, respectively. Intra-assay variability was $<9 \%$ for the 3 assays.

Supine blood pressure was assessed using a manual sphygmomanometer. After a $10 \mathrm{~min}$ rest period, blood pressure was measured 3 times and the average measurements used for statistical analyses.

cf-PWV measurements were obtained under the same conditions, including constant room temperature of $19^{\circ} \mathrm{C}$ to $21{ }^{\circ} \mathrm{C}$. We determined cf-PWV using a SphygmoCor automatic device (AtCor Medical, Sydney, Australia). SphygmoCor offers the possibility of cf-PWV measurements in two steps [19]. The first step is simultaneous recording of carotid pulse wave and ECG, and the second is recording of the femoral pulse wave and ECG. ECG recording during measurements is necessary for synchronization of carotid and femoral pulse wave times. Transit time between carotid and femoral pressure waves was calculated using the foot-to-foot method. The foot of the wave was identified using intersecting tangent 
algorithms. An abnormal cf-PWV was defined as standard cf-PWV that is superior to the decade-specific 90th percentile of age for normal subjects.

\section{Statistical analysis}

The statistical software package SPSS18.0 (SPSS Inc., Chicago, IL, USA) was employed. Continuous variables in normal distribution were compared using Student's t-test and analysis of variance (ANOVA). Categorical variables were analyzed using the chi-squared test. Patients were divided into 2 groups according to their gender and hypertension or cf-PWV progression. In this study, we defined cf-PWV $>12 \mathrm{~m} / \mathrm{s}$ as a clinically significant outlier [20]. Subject with baseline cf-PWV $<10 \mathrm{~m} / \mathrm{s}$ and follow-up cf$\mathrm{PWV}>12 \mathrm{~m} / \mathrm{s}$ was regarded as PWV progression. In order to assess the difference between baseline PWV, follow-up PWV and $\triangle \mathrm{PWV}$ (change from baseline PWV to follow-up PWV) with hypertension and PWV progression, we used generalized linear model (GLM) after adjusting for covariates. Partial Spearman correlation coefficients were used to clarify the association between PWV parameters and cholesterol components or ratios. To assess the independent value of sdLDL-C and different cholesterol ratios in predicting the progression of arterial stiffness, we used multivariate linear regression analysis models. We used the multivariate Cox regression model to analyze the predictive value of these ratios and sdLDL-C for cf-PWV progression. Cox proportional hazards model was used to evaluate the relative risk of progression of arteriosclerosis between sdLDL-C and TG/HDL-C three quantile subgroups. The data are expressed as the mean $\pm \mathrm{SD}$. $p$ values $<0.05$ were considered statistically significant.

\section{Results}

A total of 816 consecutive subjects that met the inclusion criteria were enrolled and completed follow-up. The participant population included 476 (58.3\%) men, and the average age of participants was $39.1 \pm 7.8$ years. Baseline blood pressure parameters were as follows: SBP $(125.9 \pm 9.4 \mathrm{mmHg}), \mathrm{DBP}(75.2 \pm 7.1 \mathrm{mmHg})$, pulse pressure (PP) $(50.7 \pm 9.5 \mathrm{mmHg})$ and MAP $(92.1 \pm 6.5 \mathrm{~mm}$ $\mathrm{Hg}$ ). Baseline mean cf-PWV was $9.90 \pm 0.84 \mathrm{~m} / \mathrm{s}$ and follow-up was $10.51 \pm 1.12 \mathrm{~m} / \mathrm{s}$. The annualized average progression of cf-PWV was $0.12 \pm 0.08 \mathrm{~m} / \mathrm{s} /$ year. After 5 years of follow-up, 129 normal blood pressure subjects developed hypertension. Among them, grade Ihypertensive patients were 82 , grade II were 38 and grade III were 9. In addition, 60 subjects used drugs to control blood pressure. At the end of follow-up, the cf-PWV of hypertensions were significantly higher than normal subjects $(11.17 \pm 1.43$ vs $10.46 \pm 1.10 \mathrm{~m} / \mathrm{s}, p<0.001)$. At the end of the follow-up, there were a total of 144 patients with cf-PWV progression, with an average of $12.70 \pm$ $0.64 \mathrm{~m} / \mathrm{s}$.
We divided all subjects into two groups according to their gender. Male subjects were more likely to smoke and drink than female subjects. However, educational attainment, family income and physical activity were no difference between male and female. Males had significantly higher levels of age $(40.5 \pm 7.4$ vs $37.2 \pm 8.1, \mathrm{p}<0.001)$, BMI $(24.8 \pm 2.3$ vs $21.0 \pm 2.2$, p $<0.001$ ), blood urea nitrogen (BUN, $5.18 \pm 1.23$ vs $4.99 \pm 1.13, p=0.021)$, creatinine $(\mathrm{Cr}, 68.4 \pm 10.0$ vs $66.7 \pm 10.4, p=0.016)$, sdLDL-C $(0.72 \pm 0.28$ vs $0.66 \pm$ $0.26, p=0.004)$, sdLDL-C/HDL-C ratio $(0.64 \pm 0.35$ vs $0.58 \pm 0.29, p=0.011)$, baseline PWV $(9.96 \pm 0.84$ vs $9.83 \pm 0.85, p=0.03)$, follow-up PWV $(10.66 \pm 1.19$ vs $10.45 \pm 1.18, \mathrm{p}=0.01), \quad \triangle \mathrm{PWV}(0.70 \pm 0.55$ vs $0.62 \pm$ $0.48, \mathrm{p}=0.02)$ and hs-CRP $(0.85 \pm 0.21$ vs $0.64 \pm 0.16$, $\mathrm{p}=0.004$ ) than females (Table 1). Table 2 showed the difference of PWV parameters, cholesterol components and ratios between PWV, hypertension progression subjects with non-progression subjects. PWV progression subjects had higher levels of baseline PWV, follow-up PWV, $\triangle$ PWV, TG, sdLDL-C and TG/ HDL-C ratio. After adjusting for covariates such as age, gender, BMI, FBG, TNF- $\alpha$, IL- 6 and hs-CRP, GLM analyses showed that subjects with PWV and hypertension progression had higher baseline PWV, follow-up PWV and $\triangle \mathrm{PWV}$ than those with no PWV and hypertension progression. Meanwhile, GLM analyses also showed subjects with 90th percentile age had higher baseline PWV, follow-up PWV and $\triangle \mathrm{PWV}$ compared with the other percentile subjects $(p<0.001$, Fig. 1).

Table 3 gave partial correlation coefficients between PWV parameters and cholesterol components, ratios and inflammation factors after adjustment for age, gender, BMI, exercise, smoking, drinking, SBP, MAP, FBG and UA. Partial Spearman correlation analysis showed that sdLDL-C, TG/HDL-C and IL-6 were correlated weakly but significantly with baseline PWV. sdLDL-C/ HDL-C had a weakly correlation with follow-up PWV and $\triangle \mathrm{PWV}$. Both sdLDL-C and TG/HDL-C were significantly correlated with all PWV parameters. Meanwhile, Pearson analysis also confirmed a positive correlation between sdLDL-C and TG/HDL-C $(\mathrm{r}=0.433, \mathrm{p}<0.001)$.

Then, the associations of sdLDL-C and cholesterol ratios with the PWV parameters were adjusted for covariates using multiple regression models. As shown in Table 4. sdLDL-C was the most closely associated with follow-up PWV and $\triangle \mathrm{PWV}$ even after multivariate adjustment. The adjusted regression coefficients ( $\beta$ values) were 0.222 for follow-up PWV, 0.275 for $\triangle \mathrm{PWV}$. However, sdLDL-C and baseline PWV did not show significant association in the multiple regression models $(\beta=0.192, p=0.065)$. Homoplastically, sdLDL$\mathrm{C} / \mathrm{HDL}-\mathrm{C}$ had a similar statistical result to sd LDL-C, 
Table 1 Clinical and laboratory data for 816 normotensive subjects divided into two groups according to their gender

\begin{tabular}{|c|c|c|c|c|}
\hline Variables & Men $(n=476)$ & Women $(n=340)$ & $\begin{array}{l}\text { All subjects } \\
(n=816)\end{array}$ & $p$ value \\
\hline Age(years) & $40.5 \pm 7.4$ & $37.2 \pm 8.1$ & $39.1 \pm 7.8$ & $<0.001$ \\
\hline Education(years) & & & & 0.211 \\
\hline $\operatorname{Low}(<9 \mathrm{y})$ & $70(14.7 \%)$ & $64(18.8 \%)$ & 134(16.4\%) & \\
\hline Middle(9-12y) & 178(37.4\%) & $120(35.3 \%)$ & $298(36.5 \%)$ & \\
\hline $\operatorname{High}(>12 y)$ & $228(47.9 \%)$ & $156(45.9 \%)$ & $384(47.1 \%)$ & \\
\hline Family income(CNY/y) & & & & 0.683 \\
\hline$<30,000$ & $82(17.3 \%)$ & $59(17.4 \%)$ & $141(17.3 \%)$ & \\
\hline $30,000-100,000$ & $274(57.7 \%)$ & $187(55.0 \%)$ & $461(56.6 \%)$ & \\
\hline$>100,000$ & $119(25.0 \%)$ & $94(27.6 \%)$ & 213(26.1\%) & \\
\hline Physical activity & & & & 0.760 \\
\hline Low & $200(42.0 \%)$ & $155(45.6 \%)$ & $355(43.5 \%)$ & \\
\hline Intermediate & 178(37.4\%) & $112(32.9 \%)$ & $290(35.5 \%)$ & \\
\hline High & $98(20.6 \%)$ & $73(21.5 \%)$ & $171(21.0 \%)$ & \\
\hline $\mathrm{BMI}\left(\mathrm{kg} / \mathrm{m}^{2}\right)$ & $24.8 \pm 2.3$ & $21.0 \pm 2.2$ & $23.2 \pm 2.9$ & $<0.001$ \\
\hline Smoking (\%) & 163(34.2\%) & 13(3.8\%) & $176(21.6 \%)$ & $<0.001$ \\
\hline Drinking (\%) & $139(29.2 \%)$ & 19(5.6\%) & 158(19.4\%) & $<0.001$ \\
\hline $\mathrm{SBP}(\mathrm{mm} \mathrm{Hg})$ & $126.2 \pm 9.2$ & $125.4 \pm 9.6$ & $126.0 \pm 9.4$ & 0.245 \\
\hline $\mathrm{DBP}(\mathrm{mm} \mathrm{Hg})$ & $75.0 \pm 7.1$ & $75.5 \pm 7.1$ & $75.2 \pm 7.1$ & 0.344 \\
\hline $\mathrm{PP}(\mathrm{mm} \mathrm{Hg})$ & $51.2 \pm 9.5$ & $49.9 \pm 9.6$ & $50.7 \pm 9.5$ & 0.065 \\
\hline $\mathrm{MAP}(\mathrm{mm} \mathrm{Hg})$ & $92.0 \pm 6.5$ & $92.1 \pm 6.6$ & $92.0 \pm 6.5$ & 0.827 \\
\hline $\mathrm{HR}(\mathrm{bpm})$ & $72.6 \pm 7.2$ & $71.7 \pm 7.3$ & $72.2 \pm 7.2$ & 0.083 \\
\hline $\mathrm{ALT}(\mathrm{U} / \mathrm{L})$ & $26.5 \pm 15.9$ & $28.0 \pm 16.4$ & $27.1 \pm 16.1$ & 0.196 \\
\hline AST(U/L) & $22.8 \pm 11.3$ & $22.1 \pm 9.5$ & $22.5 \pm 10.6$ & 0.391 \\
\hline $\mathrm{GGT}(\mathrm{U} / \mathrm{L})$ & $29.6 \pm 18.4$ & $29.5 \pm 17.7$ & $29.6 \pm 18.1$ & 0.957 \\
\hline $\mathrm{BUN}(\mathrm{mmol} / \mathrm{L})$ & $5.18 \pm 1.23$ & $4.99 \pm 1.13$ & $5.10 \pm 1.19$ & 0.021 \\
\hline $\mathrm{Cr}$ (ummol/L) & $68.4 \pm 10.0$ & $66.7 \pm 10.4$ & $67.7 \pm 10.2$ & 0.016 \\
\hline UA(ummol/L) & $290.8 \pm 93.1$ & $291.9 \pm 94.8$ & $291.3 \pm 93.7$ & 0.874 \\
\hline $\mathrm{FBG}(\mathrm{mmol} / \mathrm{L})$ & $5.11 \pm 0.61$ & $5.05 \pm 0.61$ & $5.09 \pm 0.61$ & 0.186 \\
\hline $\mathrm{TC}(\mathrm{mmol} / \mathrm{L})$ & $4.81 \pm 0.77$ & $4.76 \pm 0.79$ & $4.79 \pm 0.78$ & 0.466 \\
\hline $\mathrm{TG}(\mathrm{mmol} / \mathrm{L})$ & $1.68 \pm 0.72$ & $1.62 \pm 0.73$ & $1.65 \pm 0.73$ & 0.259 \\
\hline $\mathrm{LDL}-\mathrm{C}(\mathrm{mmol} / \mathrm{L})$ & $3.00 \pm 0.76$ & $2.95 \pm 0.77$ & $2.98 \pm 0.76$ & 0.349 \\
\hline $\mathrm{HDL}-\mathrm{C}(\mathrm{mmol} / \mathrm{L})$ & $1.21 \pm 0.30$ & $1.20 \pm 0.26$ & $1.21 \pm 0.29$ & 0.844 \\
\hline sdLDL-C(mmol/L) & $0.72 \pm 0.28$ & $0.66 \pm 0.26$ & $0.70 \pm 0.27$ & 0.004 \\
\hline $\mathrm{ApoB}(\mathrm{g} / \mathrm{L})$ & $0.95 \pm 0.26$ & $0.94 \pm 0.25$ & $0.94 \pm 0.26$ & 0.390 \\
\hline ApoA-1(g/L) & $1.21 \pm 0.31$ & $1.24 \pm 0.30$ & $1.23 \pm 0.31$ & 0.102 \\
\hline $\mathrm{TC} / \mathrm{HDL}-\mathrm{C}$ & $4.21 \pm 1.25$ & $4.14 \pm 1.16$ & $4.18 \pm 1.21$ & 0.434 \\
\hline TG/HDL-C & $1.48 \pm 0.79$ & $1.39 \pm 0.68$ & $1.44 \pm 0.75$ & 0.12 \\
\hline LDL-C/HDL-C & $2.64 \pm 0.95$ & $2.57 \pm 0.90$ & $2.61 \pm 0.93$ & 0.312 \\
\hline sdLDL-C/HDL-C & $0.64 \pm 0.35$ & $0.58 \pm 0.29$ & $0.62 \pm 0.33$ & 0.011 \\
\hline ApoB/ApoA-1 & $0.81 \pm 0.32$ & $0.82 \pm 0.30$ & $0.82 \pm 0.31$ & 0.687 \\
\hline Baseline PWV(m/s) & $9.96 \pm 0.84$ & $9.83 \pm 0.85$ & $9.91 \pm 0.84$ & 0.03 \\
\hline Follow-up PWV(m/s) & $10.66 \pm 1.19$ & $10.45 \pm 1.18$ & $10.57 \pm 1.19$ & 0.01 \\
\hline$\triangle \mathrm{PW}(\mathrm{m} / \mathrm{s})$ & $0.70 \pm 0.55$ & $0.62 \pm 0.48$ & $0.67 \pm 0.52$ & 0.02 \\
\hline
\end{tabular}


Table 1 Clinical and laboratory data for 816 normotensive subjects divided into two groups according to their gender (Continued)

\begin{tabular}{lllll}
\hline Variables & Men $(n=476)$ & Women $(n=340)$ & $\begin{array}{l}\text { All subjects } \\
(n=816)\end{array}$ & $p$ value \\
\hline PWV progression (\%) & $93(19.5 \%)$ & $51(15.0 \%)$ & $144(17.6 \%)$ & 0.094 \\
HT progression (\%) & $82(17.2 \%)$ & $47(13.8 \%)$ & $129(15.8 \%)$ & 0.189 \\
hs-CRP(mg/L) & $0.85 \pm 0.21$ & $0.64 \pm 0.16$ & $0.76 \pm 0.19$ & 0.004 \\
TNF-a $(\mathrm{pg} / \mathrm{ml})$ & $3.03 \pm 0.83$ & $3.04 \pm 0.81$ & $3.04 \pm 0.82$ & 0.974 \\
IL-6(pg/ml) & $2.70 \pm 1.17$ & $2.60 \pm 1.15$ & $2.66 \pm 1.17$ & 0.234 \\
\hline
\end{tabular}

accordingly for baseline PWV $(\beta=0.034, p=0.697)$, follow-up PWV $(\beta=0.128, p=0.009)$ and $\triangle \mathrm{PWV}(\beta=$ 0.137, $p=0.011)$. TG/HDL-C was only one cholesterol ratios that associated with all PWV parameters in the multiple regression models.

Further analyses were performed to examine sdLDL-C and cholesterol ratios that were associated with PWV progression. At the end of an average of 5 years of follow-up, 144 subjects had PWV progression. We used the Cox regression model to analyze the predictive value of these cholesterol ratios and sdLDL-C for cf-PWV progression. After adjusted the covariates including gender, age, exercise, smoking, drinking, BMI, SBP, MAP, FBG, UA, baseline
PWV, TNF- $\alpha$, hs-CPR and IL-6, in our study population, sdLDL-C and TG/HDL-C were able to independently predict PWV progression after 5 years (Table 5). It seems that sdLDL-C had higher predicting power than TG/HDL-C (unadjusted: 2.460 vs 1.414; adjusted: 2.070 vs 1.355 ).

The subjects were grouped based on the tri-sectional quantiles of the sdLDL-C level and TG/HDL-C ratio. TG/HDL-C tri-sectional quantile group was $<1.05$, $1.05-1.65$, and $>1.65$ and sdLDL-C tri-sectional quantile group was $\leq 0.55 \mathrm{mmol} / \mathrm{L}, \quad 0.56-0.81 \mathrm{mmol} / \mathrm{L}$ and $\geq 0.82 \mathrm{mmol} / \mathrm{L}$. Cox risk regression model analysis showed that the risk of arterial stiffness progression in the subjects with high sd-LDL-C quantiles were

Table 2 PWV parameters, cholesterol parameters and ratios and inflammatory factors of subjects by PWV and hypertension progression

\begin{tabular}{|c|c|c|c|c|c|c|}
\hline Variables & non-PW progression & PWV progression & $p$ value & non-HT progression & HT progression & $p$ value \\
\hline Age(years) & $38.5 \pm 7.7$ & $41.8 \pm 7.7$ & $<0.001$ & $38.6 \pm 8.2$ & $41.7 \pm 5.2$ & $<0.001$ \\
\hline Baseline PWV(m/s) & $9.63 \pm 0.58$ & $11.18 \pm 0.71$ & $<0.001$ & $9.89 \pm 0.79$ & $9.99 \pm 0.98$ & 0.092 \\
\hline Follow-up PWV(m/s) & $10.12 \pm 0.67$ & $12.70 \pm 0.64$ & $<0.001$ & $10.46 \pm 1.10$ & $11.17 \pm 1.43$ & $<0.001$ \\
\hline$\Delta \mathrm{PW}(\mathrm{m} / \mathrm{s})$ & $0.48 \pm 0.27$ & $1.52 \pm 0.56$ & $<0.001$ & $0.63 \pm 0.49$ & $0.87 \pm 0.62$ & $<0.001$ \\
\hline$\triangle \mathrm{SBP}(\mathrm{mmHg})$ & $3.11 \pm 0.78$ & $3.38 \pm 0.85$ & 0.205 & $2.75 \pm 0.74$ & $3.86 \pm 0.81$ & $<0.01$ \\
\hline$\triangle \mathrm{DBP}(\mathrm{mmHg})$ & $2.82 \pm 0.69$ & $3.63 \pm 0.77$ & 0.039 & $2.79 \pm 0.58$ & $3.81 \pm 0.80$ & $<0.01$ \\
\hline$\Delta \mathrm{PP}(\mathrm{mmHg})$ & $0.26 \pm 0.08$ & $0.27 \pm 0.06$ & 0.765 & $0.25 \pm 0.09$ & $0.28 \pm 0.10$ & 0.113 \\
\hline$\triangle \mathrm{MAP}(\mathrm{mmHg})$ & $3.08 \pm 0.57$ & $3.72 \pm 0.61$ & 0.032 & $2.88 \pm 0.45$ & $3.79 \pm 0.57$ & $<0.01$ \\
\hline $\mathrm{TC}(\mathrm{mmol} / \mathrm{L})$ & $4.81 \pm 0.80$ & $4.69 \pm 0.67$ & 0.086 & $4.80 \pm 0.79$ & $4.75 \pm 0.75$ & 0.515 \\
\hline $\mathrm{TG}(\mathrm{mmol} / \mathrm{L})$ & $1.58 \pm 0.69$ & $1.96 \pm 0.83$ & $<0.001$ & $1.64 \pm 0.72$ & $1.72 \pm 0.81$ & 0.276 \\
\hline $\mathrm{LDL}-\mathrm{C}(\mathrm{mmol} / \mathrm{L})$ & $2.98 \pm 0.79$ & $2.96 \pm 0.63$ & 0.684 & $2.99 \pm 0.77$ & $2.95 \pm 0.70$ & 0.566 \\
\hline $\mathrm{HDL}-\mathrm{C}(\mathrm{mmol} / \mathrm{L})$ & $1.20 \pm 0.28$ & $1.22 \pm 0.29$ & 0.352 & $1.21 \pm 0.28$ & $1.23 \pm 0.32$ & 0.469 \\
\hline sdLDL-C(mmol/L) & $0.68 \pm 0.28$ & $0.77 \pm 0.25$ & $<0.001$ & $0.70 \pm 0.27$ & $0.69 \pm 0.31$ & 0.661 \\
\hline ApoB(g/L) & $0.94 \pm 0.25$ & $0.96 \pm 0.27$ & 0.301 & $0.94 \pm 0.25$ & $0.96 \pm 0.28$ & 0.516 \\
\hline ApoA-1 (g/L) & $1.23 \pm 0.30$ & $1.23 \pm 0.33$ & 0.916 & $1.23 \pm 0.30$ & $1.21 \pm 0.32$ & 0.523 \\
\hline TC/HDL-C & $4.21 \pm 1.23$ & $4.02 \pm 1.09$ & 0.081 & $4.19 \pm 1.18$ & $4.14 \pm 1.37$ & 0.709 \\
\hline TG/HDL-C & $1.39 \pm 0.72$ & $1.69 \pm 0.83$ & $<0.001$ & $1.43 \pm 0.72$ & $1.51 \pm 0.89$ & 0.233 \\
\hline LDL-C/HDL-C & $2.62 \pm 0.95$ & $2.55 \pm 0.82$ & 0.398 & $2.61 \pm 0.91$ & $2.59 \pm 1.02$ & 0.794 \\
\hline SdLDL-C/HDL-C & $0.61 \pm 0.34$ & $0.66 \pm 0.28$ & 0.063 & $0.62 \pm 0.31$ & $0.61 \pm 0.40$ & 0.857 \\
\hline ApoB/ApoA-1 & $0.81 \pm 0.31$ & $0.84 \pm 0.32$ & 0.357 & $0.81 \pm 0.31$ & $0.84 \pm 0.32$ & 0.338 \\
\hline TNF-a(pg/ml) & $3.02 \pm 0.82$ & $3.13 \pm 0.80$ & 0.153 & $3.05 \pm 0.82$ & $2.99 \pm 0.83$ & 0.492 \\
\hline IL-6(pg/ml) & $2.69 \pm 1.18$ & $2.53 \pm 1.12$ & 0.126 & $2.67 \pm 1.17$ & $2.63 \pm 1.18$ & 0.738 \\
\hline hs-CRP(mg/L) & $0.74 \pm 0.19$ & $0.84 \pm 0.22$ & 0.298 & $0.75 \pm 0.16$ & $0.84 \pm 0.21$ & 0.350 \\
\hline
\end{tabular}




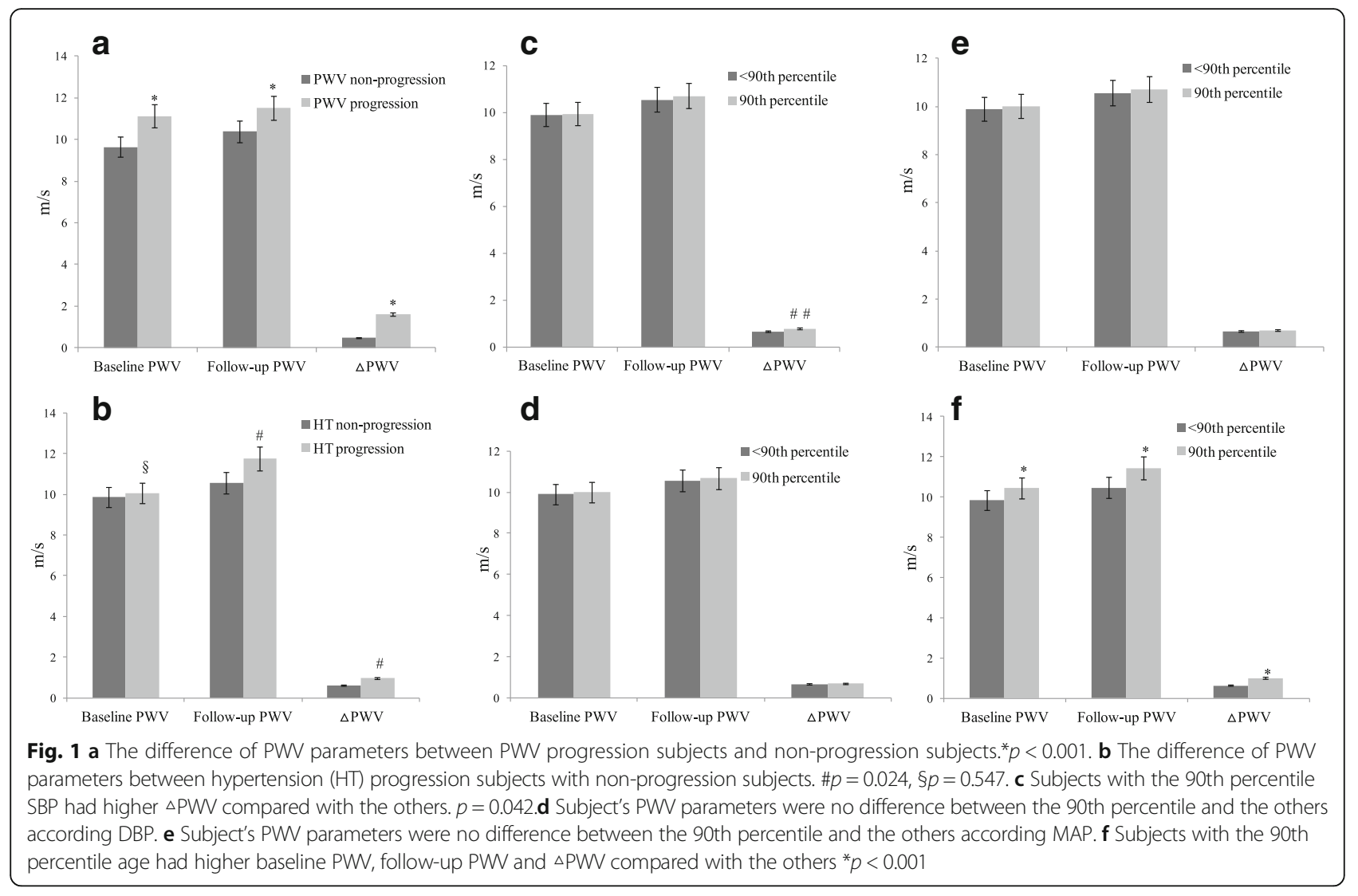

Table 3 Partial correlation coefficients between PWV parameters with cholesterol parameters and ratios and inflammatory factors

\begin{tabular}{llll}
\hline All subjects $(n=816)$ & Baseline PW, r(p)a & Follow-up PW, r(p)b & $\Delta$ PW, r(p)a \\
\hline TC & $0.022(0.539)$ & $0.038(0.276)$ & $0.051(0.149)$ \\
TG & $0.019(0.149)$ & $0.022(0.229)$ & $0.029(0.209)$ \\
LDL-C & $0.001(0.970)$ & $0.004(0.903)$ & $0.007(0.833)$ \\
HDL-C & $-0.058(0.101)$ & $-0.044(0.212)$ & $-0.005(0.882)$ \\
sdLDL-C & $0.064(0.07)$ & $0.113(0.001)$ & $0.149(<0.001)$ \\
ApoB & $0.009(0.802)$ & $0.016(0.656)$ & $0.021(0.556)$ \\
ApoA-1 & $-0.058(0.099)$ & $-0.043(0.219)$ & $-0.003(0.932)$ \\
TC-C/HDL-C & $-0.056(0.112)$ & $-0.059(0.093)$ & $-0.042(0.233)$ \\
TG/HDL-C & $0.092(0.009)$ & $0.152(<0.001)$ & $0.191(<0.001)$ \\
LDL-C/HDL-C & $-0.029(0.406)$ & $-0.022(0.525)$ & $-0.003(0.933)$ \\
sdLDL-C/HDL-C & $0.013(0.704)$ & $0.050(0.05)$ & $0.09(0.01)$ \\
ApoB/ApoA-1 & $0.040(0.263)$ & $0.042(0.233)$ & $0.030(0.389)$ \\
TNF-a & $0.022(0.534)$ & $0.030(0.393)$ & $0.032(0.366)$ \\
IL-6 & $0.090(0.011)$ & $0.076(0.031)$ & $0.025(0.481)$ \\
hs-CRP & $0.010(0.783)$ & $0.006(0.869)$ & $-0.003(0.942)$ \\
\hline
\end{tabular}

Model a was adjusted for gender, age, physical activity, smoking, drinking, BMI, SBP, MAP, FBG and UA Model $\mathrm{b}$ was adjusted for a plus baseline PWV 
Table 4 Univariable and multivariable linear regression of SdLDL-C and cholesterol ratios with PWV parameters

\begin{tabular}{|c|c|c|c|c|c|c|}
\hline \multirow{2}{*}{$\frac{\text { Variables }}{\text { sdLDL-C }}$} & \multicolumn{2}{|c|}{ Baseline PWV $\beta$-Coefficient $p$ value } & \multicolumn{2}{|c|}{ Follow-up PWV $\beta$-Coefficient $p$ value } & \multicolumn{2}{|c|}{$\triangle \mathrm{PWV} \beta$-Coefficient $p$ value } \\
\hline & & & & & & \\
\hline unadjusted & 0.290 & 0.007 & 0.623 & $<0.001$ & 0.333 & $<0.001$ \\
\hline adjusted & $0.192 \mathrm{a}$ & 0.065 & $0.222 b$ & $<0.001$ & $0.275 \mathrm{a}$ & $<0.001$ \\
\hline \multicolumn{7}{|l|}{$\mathrm{TC} / \mathrm{HDL}-\mathrm{C}$} \\
\hline unadjusted & -0.036 & 0.145 & -0.053 & 0.123 & -0.017 & 0.247 \\
\hline adjusted & $-0.044 \mathrm{a}$ & 0.061 & $-0.007 b$ & 0.577 & $-0.019 a$ & 0.189 \\
\hline \multicolumn{7}{|l|}{$\mathrm{TG} / \mathrm{HDL}-\mathrm{C}$} \\
\hline unadjusted & 0.126 & 0.001 & 0.267 & $<0.001$ & 0.141 & $<0.001$ \\
\hline adjusted & $0.095 \mathrm{a}$ & 0.012 & $0.102 b$ & $<0.001$ & 0.127 a & $<0.001$ \\
\hline \multicolumn{7}{|l|}{ LDL-C/HDL-C } \\
\hline unadjusted & -0.025 & 0.442 & -0.026 & 0.568 & -0.001 & 0.955 \\
\hline adjusted & $-0.032 \mathrm{a}$ & 0.302 & $0.005 b$ & 0.753 & $-0.003 \mathrm{a}$ & 0.870 \\
\hline \multicolumn{7}{|l|}{ sdLDL-C/HDL-C } \\
\hline unadjusted & 0.100 & 0.269 & 0.275 & 0.031 & 0.175 & 0.002 \\
\hline adjusted & $0.034 \mathrm{a}$ & 0.697 & $0.128 b$ & 0.009 & $0.137 \mathrm{a}$ & 0.011 \\
\hline \multicolumn{7}{|l|}{ ApoB/ApoA-1 } \\
\hline unadjusted & 0.070 & 0.462 & 0.103 & 0.439 & 0.034 & 0.567 \\
\hline adjusted & $0.107 a$ & 0.239 & $0.022 b$ & 0.667 & $0.051 \mathrm{a}$ & 0.369 \\
\hline
\end{tabular}

Model a was adjusted for gender, age, physical activity, smoking, drinking, BMI, SBP, MAP, FBG, UA, TNF-a, hs-CPR and IL-6 Model $b$ was adjusted for a plus baseline PWV

Table 5 Cox proportional hazards model for PWV progression ${ }^{a}$

\begin{tabular}{|c|c|c|c|}
\hline Variables & OR & $95 \% \mathrm{Cl}$ & $p$ value \\
\hline \multicolumn{4}{|l|}{ sdLDL-C } \\
\hline unadjusted & 2.460 & 1.400 to 4.321 & 0.002 \\
\hline adjusted & 2.070 & 1.162 to 3.688 & 0.014 \\
\hline \multicolumn{4}{|l|}{$\mathrm{TC} / \mathrm{HDL}-\mathrm{C}$} \\
\hline unadjusted & 0.873 & 0.754 to 1.012 & 0.071 \\
\hline adjusted & 0.954 & 0.819 to 1.110 & 0.541 \\
\hline \multicolumn{4}{|l|}{ TG/HDL-C } \\
\hline unadjusted & 1.414 & 1.185 to 1.687 & $<0.001$ \\
\hline adjusted & 1.355 & 1.136 to 1.617 & 0.001 \\
\hline \multicolumn{4}{|l|}{ LDL-C/HDL-C } \\
\hline unadjusted & 0.924 & 0.769 to 1.110 & 0.396 \\
\hline adjusted & 0.896 & 0.742 to 1.082 & 0.253 \\
\hline \multicolumn{4}{|l|}{ sdLDL-C/HDL-C } \\
\hline unadjusted & 1.379 & 0.877 to 2.168 & 0.165 \\
\hline adjusted & 1.255 & 0.780 to 2.020 & 0.350 \\
\hline \multicolumn{4}{|l|}{ ApoB/ApoA-1 } \\
\hline unadjusted & 1.178 & 0.715 to 1.941 & 0.521 \\
\hline adjusted & 1.294 & 0.786 to 2.129 & 0.311 \\
\hline
\end{tabular}

a Adjusted for gender, age, physical activity, smoking, drinking, BMI, SBP, MAP, FBG, UA, baseline PWV, TNF-a, hs-CPR and IL-6 significantly higher than those with low quantiles at the end of the follow-up period (Tertiles 3 vs Tertiles1, RR $=2.867,95 \%$ CI: 1.106 to $7.434, p=0.03)$. Meanwhile, the risk of high TG/HDL-C ratio quantiles was significantly higher than low quantiles (Tertiles 3 vs Tertiles1, RR = 2.051, 95\%CI:1.105 to 3.807, $p=0.023$ ). However, there was no significant difference between the sdLDL-C and TG/HDL-C ratio median quantiles and low quantiles subjects with arterial stiffness risk (Fig. 2).

\section{Discussion}

Based on the Framingham Heart Study published in 1959, cholesterol levels were the first confirmed cardiovascular risk factor [21]. Currently, it is understood that blood lipid parameters and early atherosclerosis [22], CIMT [12, 14] and arterial stiffness [23] are connected. sdLDL-C was considered as the best marker for predicting carotid atherosclerosis [11]. However, the association between sdLDL-C and arterial stiffness was still unclear. We systematically and simultaneously evaluate the effects of sdLDL-C and cholesterol ratios on arterial stiffness progression in normal blood pressure subjects. With an average of 5 years follow-up, we found that sdLDL-C and TG/HDL-C ratio could independently predict arterial stiffness progression after adjusting for other cardiovascular risk factors. Subjects with a high sdLDL$\mathrm{C}$ level and TG/HDL-C ratio had a higher risk of arterial stiffness. 

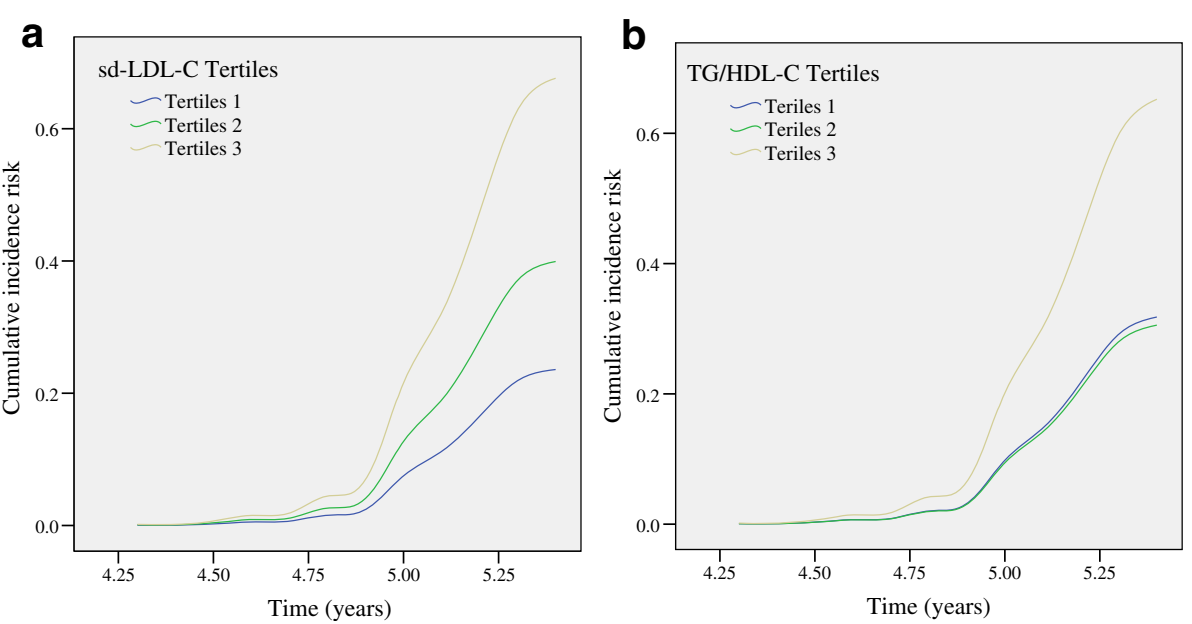

Fig. 2 Cox risk regression model analysis showed that the relative risk of arterial stiffness progression in the different tertiles groups of sd-LDL-C and TG/HDL-C ratio

Previous studies showed that sdLDL-C was the best marker for predicting carotid arteriosclerosis by using CAIMT [11]. sdLDL-C was a quantitative risk marker of arteriosclerosis than the standard lipid parameters [10]. The key finding of the present study was further confirmed that sdLDL-C was a better lipid variable than other cholesterol ratios in assessing the risk of arterial stiffness progression using cf-PWV. sdLDL-C may be the best indicator for predicting arterial stiffness in all lipid parameters and cholesterol ratios. There are several explanations for this finding. First, as compared with the larger size counterparts, sdLDL-C particles have lower affinity to LDL receptors [24], and have longer residence time in the circulation [25]. Second, due to sdLDL-C particles are small and have larger specific surface area, they are easier penetration into arterial wall [26]. Third, sdLDL-C is one of the most atherogenic lipoprotein classes and higher susceptibility to oxidative modification [27]. Finally, sdLDL-C level may be an integrated marker for atherogenic risk $[10,28]$. sdLDL-C was strongly correlated with TG, HDL-C and apoA-1 [11]. In our healthy population, the baseline sdLDL-C levels of PWV progression subjects were significantly higher than non progressive subjects. sdLDL-C can predict progression of arterial stiffness progression independently with other cardiovascular risk factors. However, sdLDL-C levels were not associated with baseline PWV. These results show that sdLDL-C gradually plays a role in promoting arterial stiffness progression.

In our normal blood pressure population, high TG/ HDL-C ratio subjects have a higher risk of arterial stiffness. This may be associated with the fact that TG/ HDL-C can better predict sdLDL-C, which is a kind of atherosclerotic lipid particle that can strongly predict CVD [10]. Under the effect of cholesterol ester transfer protein, higher TG levels correlate with more active lipid exchanges, resulting in increased sdLDL-C concentrations and decreased HDL-C levels, which ultimately enhance arteriosclerosis-inducing factors [29]. King et al. indicated that when the cut-off point for TG/HDL-C was 1.4 , specificity and sensitivity reached $79 \%$ and $80 \%$, respectively, when using this index to predict sdLDL-C, and thus it is the best known predicator [30]. In our population, the average TG/HDL-C ratio was $1.44 \pm$ 0.75, whereas the ratio of PWV progression subjects was significantly higher than non-progression $(1.69 \pm 0.83$ vs $1.39 \pm 0.72, p<0.001)$. Meanwhile, TG/HDL-C ratio and sdLDL-C also showed a significant positive correlation. Interestingly, TG/HDL-C was associated with baseline PWV, whereas sdLDL-C and sdLDL-C/HDL-C were not correlated with it.

Previously, a limited number of studies have addressed the relation between ApoB and arterial PWV. In relatively small study populations, using multiple linear logistic regression models found that ApoB and PWV had independent positive associations after adjusting for cardiovascular risk factors [31]. In addition, $A p o B$ and the ApoB/ApoA-1 ratio were found to predict PWV assessed 6 years later [32]. However, in our population, ApoB/ ApoA-1 ratio has no association with PWV change and the follow-up PWV after 5 years. ApoB levels reflect the total number of atherogenic particles (very lowdensity lipoprotein [VLDL], VLDL remnants, LDL, lipoprotein [a]). However, it is influenced by genes and its level has an obvious difference in various ethnic groups [33]. For instance, ApoB levels in China's healthy population are significantly lower than in India. PWV is also affected by genes [34].

Many studies have suggested that arteriosclerosis was an inflammatory reaction secondary to vascular injury, 
and inflammatory factors have a sustained effect on whole process of arteriosclerosis. In healthy individuals, acute systemic inflammation increases arterial stiffness and decreases wave reflections [35]. Azra Mahmud also founded that arterial stiffness is related to systemic inflammation including TNF- $\alpha$, IL- 6 and hs-CRP in essential hypertension [36]. We measured TNF- $\alpha$, IL- 6 and hs-CRP levels in our healthy subjects at baseline. After adjusted cardiovascular risk factors, partial Spearman correlation analysis showed that only IL-6 was correlated weakly with baseline and follow-up PWV. Both sdLDL$\mathrm{C}$ and TG/HDL-C are able to independently predict arterial stiffness progression after adjusted cardiovascular risk factors and inflammatory factors. High level of sdLDL-C and disproportion of TG and HDL-C may be an initiating factor to promote arterial stiffness progression in healthy.

This study has several limitations. Dyslipidemia in the Chinese population is mainly type IV hyperlipidemia, characterized by hypertriglyceridemia and low HDL-C, which is closely related to the carbohydrate-based diet in the general Chinese population [37]. Additionally, more subjects included in the study accepted tests in the cold season, and the temperature change may have affected the dietary structure of the population, resulting in increased variability of lipid components. Although this study is a multicenter study, however, it is not a multiethnic study. It is important to clarify that genes have an effect on PWV and sdLDL-C.

\section{Conclusions}

In conclusion, our study showed that sdLDL-C and TG/ HDL-C ratio could independently predict arterial stiffness progression after adjusting for other CVD risk factors. Subjects with a high sdLDL-C level and TG/ HDL-C ratio had a higher risk of arterial stiffness. However, it remains to be confirmed whether these conclusions can be applied to other populations.

\footnotetext{
Abbreviations

$\triangle \mathrm{PWV}$ : Change from baseline PWV to follow-up PWV; ALT: Alanine aminotransferase; Apo B: Apolipoproteins B; ApoA-1: Apolipoproteins A-1; AST: Aspartate aminotransferase; BMI: Body mass index; BUN: Blood urea nitrogen; CAVI: Cardio-ankle vascular index; cf-PWV: Carotid-femoral pulse wave velocity; CIMT: Carotid intima media thickness; Cr: Creatinine; CVD: Cardiovascular disease; DBP: Diastolic blood pressure; FBG: Fasting blood glucose; GGT: Gamma-glutamyltransferase; HDL-C: High density lipoprotein cholesterol; HR: Heart rate; hs-CRP: High-sensitivity C reactive protein; HT: Hypertension; IL-6: Interleukin-6; LDL-C: Low-density lipoprotein cholesterol; MAP: Mean arterial pressure; PP: Pulse pressure; SBP: Systolic blood pressure; sdLDL-C: Small dense low-density lipoprotein cholesterol; TC: Total cholesterol; TNF-a: Tumor necrosis factor a; UA: Uric acid
}

\section{Acknowledgments}

The authors are indebted to the other investigators, staff, and participants for expert technical assistance.

\section{Funding}

This study was supported by research grants from the Hospital Fund of Hubei Provincial Hospital of TCM.

Availability of data and materials Not applicable

\section{Authors' contributions}

GL, HKW and YCT contributed to the conception and design of the study. XWW, ZC, YM and JC recruited the subjects and performed the follow-up. GL and HKW performed the measure of cf-PWV. WQW analyzed the data and wrote the initial draft of the paper. $\mathrm{ZHZ}$ participated in its design and supervised the study. All authors contributed to the writing, reviewing, and revising of the manuscript.

\section{Ethics approval and consent to participate}

This study complied with the Declaration of Helsinki and was approved by the Hubei Provincial Hospital of Traditional Chinese Medicine ethics committee (No. 20100911).

\section{Consent for publication}

Not applicable

\section{Competing interests}

The authors declare that they have no competing interests.

\section{Publisher's Note}

Springer Nature remains neutral with regard to jurisdictional claims in published maps and institutional affiliations.

\section{Author details}

${ }^{1}$ Emergency Department, Hubei Provincial Hospital of Traditional Chinese Medicine, Wuhan 430061, China. ${ }^{2}$ Hubei Province Academy of Traditional Chinese Medicine, Wuhan, China. ${ }^{3}$ Department of Hepatology, Hubei Provincial Hospital of Traditional Chinese Medicine, Wuhan, China. ${ }^{4}$ Institute of Hepatology, Hubei Province Academy of Traditional Chinese Medicine, Wuhan, China. ${ }^{5}$ Department of Thoracic Surgery, TongJi Hospital, TongJi Medical College, Huazhong University of Science and Technology, Wuhan, China. ${ }^{6}$ Department of Cardiology, The Central Hospital of Wuhan, Wuhan, China. ${ }^{7}$ Emergency Department, Wuhan General Hospital of Guangzhou Military Command, Wuhan, China. ${ }^{8}$ Institute of Cardiology, Union Hospital, Tongji Medical College, Huazhong University of Science \& Technology, Wuhan, China.

Received: 3 January 2018 Accepted: 29 January 2018

Published online: 12 February 2018

\section{References}

1. Laurent S, Cockcroft J, Van Bortel L, Boutouyrie P, Giannattasio C, Hayoz D, et al. Expert consensus document on arterial stiffness: methodological issues and clinical applications. Eur Heart J. 2006;27:2588-605.

2. Laurent S, Boutouyrie P, Asmar R, Gautier I, Laloux B, Guize L, et al. Aortic stiffness is an independent predictor of all-cause and cardiovascular mortality in hypertensive patients. Hypertension. 2001;37:1236-41.

3. Blacher J, Asmar R, Djane S, London GM, Safar ME. Aortic pulse wave velocity as a marker of cardiovascular risk in hypertensive patients. Hypertension. 1999;33(5):1111-7.

4. Meaume S, Rudnichi A, Lynch A, Bussy C, Sebban C, Benetos A, et al. Aortic pulse wave velocity as a marker of cardiovascular disease in subjects over 70 years old. J Hypertens. 2001;19(5):871-7.

5. Liu ZR, Ting CT, Zhu SX, Yin FC. Aortic compliance in human hypertension. Hypertension. 1989;14:129-36.

6. O'Rourke MF, Hayward CS. Arterial stiffness, gender and heart rate. J Hypertens. 2003;21:487-90.

7. Sa Cunha R, Pannier B, Benetos A, Philippe S, Gérard ML, Michel MJ, et al. Association between high heart rate and high arterial rigidity in normotensive and hypertensive subjects. J Hypertens. 1997;15:1423-30.

8. Logan JG, Engler MB, Kim H. Genetic determinants of arterial stiffness. Cardiovasc Transl Res. 2015;8(1):23-43.

9. Benetos A, Adamopoulos C, Bureau JM, Temmar M, Labat C, Bean K, et al. Determinants of accelerated progression of arterial stiffness in normotensive 
subjects and in treated hypertensive subjects over a 6-year period. Circulation. 2002;105(10):1202-7.

10. Hoogeveen RC, Gaubatz JW, Sun W, Dodge RC, Crosby JR, Jiang J, et al. Small dense low-density lipoprotein-cholesterol concentrations predict risk for coronary heart disease. Arterioscler Thromb Vasc Biol. 2014;34:00.

11. Shoji T, Hatsuda S, Tsuchikura S, Shinohara K, Kimoto E, Koyama H, et al. Small dense low-density lipoprotein cholesterol concentration and carotid atherosclerosis. Atherosclerosis. 2009;202(2):582-8.

12. Shah AS, Urbina EM, Khoury PR, Kimball TR, Dolan LM. Lipids and lipoprotein ratios: contribution to carotid intima media thickness in adolescents and young adults with type 2 diabetes mellitus. J Clin Lipidol. 2013;7(5):441-5.

13. Hirano $T$, Ito $Y$, Yoshino $G$. Measurement of small dense low-density lipoprotein particles. J Atheroscler Thromb. 2005;12:67-72.

14. Shimizu Y, Nakazato M, Sekita T, Kadota K, Yamasaki H, Takamura N, et al. Association of arterial stiffness and diabetes with triglycerides-to-HDL cholesterol ratio for Japanese men: the Nagasaki Islands study. Atherosclerosis. 2013;228(2):491-5.

15. Ho Cl, Chen JY, Chen SY, Tsai YW, Weng YM, Tsao YC, et al. Relationship between TG/HDL-C ratio and metabolic syndrome risk factors with chronic kidney disease in healthy adult population. Clin Nutr. 2015;34(5):874-80.

16. Ng SW, Norton EC, Popkin BM. Why have physical activity levels declined among Chinese adults? Findings from the 1991-2006 China health and nutrition surveys. Soc Sci Med. 2009;68(7):1305-14.

17. National Institute on Alcohol Abuse and Alcoholism. Health risks and benefits of alcohol consumption. Alcohol Res Health. 2000;24:5-11.

18. Hirano T, Ito Y, Saegusa H, Yoshino G. A novel and simple method for quantification of small, dense LDL. J Lipid Res. 2003;44:2193-201.

19. Rajzer MW, Wojciechowska W, Klocek M, Palka I, Brzozowska-Kiszka M, Kawecka-Jaszcz K. Comparison of aortic pulse wave velocity measured by three techniques: Complior, SphygmoCor and Arteriograph. J Hypertens. 2008;26:2001-7.

20. Reference Values for Arterial Stiffness' Collaboration. Determinants of pulse wave velocity in healthy people and in the presence of cardiovascular risk factors: 'establishing normal and reference values'. Eur Heart J. 2010;31(19):2338-50.

21. Dawber TRKW, Kannel WB, Revotskie N, Stokes J III, Kagan A, Gordon T. Some factors associated with the development of coronary heart disease: six years' follow up experience in the Framingham study. Am J Public Health Nations Health. 1959;49:1349-56.

22. Newman WP III, Freedman DS, Voors AW, Gard PD, Srinivasan SR, Cresanta $\mathrm{JL}$, et al. Relation of serum lipoprotein levels and systolic blood pressure to early atherosclerosis. N Engl J Med. 1986;314(3):138-44.

23. Wang F, Ye P, Luo L, Xiao W, Qi L, Bian S, et al. Association of serum lipids with arterial stiffness in a population-based study in Beijing. Eur J Clin Investig. 2011;41(9):929-36.

24. Chen GC, Liu W, Duchateau P, Allaart J, Hamilton RL, Mendel CM, et al. Conformational differences in human apolipoprotein B-100 among subspecies of low density lipoproteins (LDL). Association of altered proteolytic accessibility with decreased receptor binding of LDL subspecies from hypertriglyceridemic subjects. J Biol Chem. 1994;269:29121-8.

25. Packard CJ, Demant T, Stewart JP, Bedford D, Caslake MJ, Schwertfeger G, et al. Apolipoprotein B metabolism and the distribution of VLDL and LDL subfractions. J Lipid Res. 2000:41:305-18.

26. Wang H, Wang HM, Jin QH, Cong H, Zhuang GS, Zhao JL, et al. Microchipbased small, dense low-density lipoproteins assay for coronary heart disease risk assessment. Electrophoresis. 2008;29(9):1932-41.

27. Wu J, Shi YH, Niu DM, Li HQ, Zhang CN, Wang JJ. Association among retinol-binding protein 4 , small dense LDL cholesterol and oxidized LDL levels in dyslipidemia subjects. Clin Biochem. 2012;45(9):619-22.

28. Berneis KK, Krauss RM. Metabolic origins and clinical significance of LDL heterogeneity. J Lipid Res. 2002;43:1363-79.

29. Grundy SM. Hypertriglyceridemia, insulin resistance, and the metabolic syndrome. Am J Cardiol. 1999:83(9):25-9.

30. King RI, Florkowski CM, Yeo J, Walmsley TA, Shand BI, Scott RS, et al. What is the best predictor of the atherogenic LDL subclass phenotype 'pattern B'in patients with type 2 diabetes mellitus? Ann Clin Biochem. 2011;48(2):166-9.

31. Amar J, Ruidavets JB, Chamontin B, Drouet $L$, Ferrières J. Arterial stiffness and cardiovascular risk factors in a population-based study. J Hypertens. 2001;19:381-7
32. Koivistoinen T, Hutri-Kähönen N, Juonala M, Kööbi T, Aatola H, Lehtimäki T, et al. Apolipoprotein B is related to arterial pulse wave velocity in young adults: the cardiovascular risk in young Finns study. Atherosclerosis. 2011;214(1):220-4.

33. Tsimikas S, Clopton P, Brilakis ES, Marcovina SM, Khera A, Miller ER, et al. Relationship of oxidized phospholipids on Apolipoprotein B-100 particles to race/ethnicity, Apolipoprotein (a) Isoform size, and cardiovascular risk factors. Circulation. 2009;119(13):1711-9.

34. Saha N. Serum high density lipoprotein cholesterol, apolipoprotein Al, A-Il and B levels in Singapore ethnic groups. Atherosclerosis. 1987;68(1-2):117-21.

35. Vlachopoulos C, Dima I, Aznaouridis K, Vasiliadou C, Loakeimidis N, Aggeli C, et al. Acute systemic inflammation increases arterial stiffness and decreases wave reflections in healthy individuals. Circulation. 2005;112(14):2193-200.

36. Mahmud A, Feely J. Arterial stiffness is related to systemic inflammation in essential hypertension. Hypertension. 2005;46(5):1118-22.

37. Wu JY, Duan XY, Li L, Dai F, Li YY, Li XJ, et al. Dyslipidemia in Shanghai, China. Prev Med. 2010;51(5):412-5.

\section{Submit your next manuscript to BioMed Central and we will help you at every step:}

- We accept pre-submission inquiries

- Our selector tool helps you to find the most relevant journal

- We provide round the clock customer support

- Convenient online submission

- Thorough peer review

- Inclusion in PubMed and all major indexing services

- Maximum visibility for your research

Submit your manuscript at www.biomedcentral.com/submit
(O) BioMed Central 